\title{
A Difference Scheme for Solving the Stefan Problem
}

By

Tatsuo Nogi*

It is the aim of this paper to investigate how solution of the Stefan problem can be obtained by solving a new difference scheme in a rectangular lattice and taking the limit of such solution as the mesh size of the lattice tends to zero. For simplicity we shall consider a one-phase Stefan problem of heat equation

$$
a^{2} \frac{\partial^{2} u}{\partial x^{2}}-\frac{\partial u}{\partial t}=0 \quad(a: \text { positive constant })
$$

in the region $0<t<T, 0<x<y(t)$, where the boundary conditions

$$
\begin{aligned}
& u(0, t)=f(t), \\
& u(x(t), t)=0
\end{aligned}
$$

and the initial condition

$$
u(x, 0)=\varphi(x), \quad 0<x<y(0)=l
$$

are imposed. The function $x=y(t)$ is the free boundary which is not known and is to be found together with $u(x, t)$ by the Stefan condition

$$
\dot{y}(t)=\kappa \frac{\partial u}{\partial x}(y(t), t) \quad(\kappa: \text { const. }) .
$$

With the heat equation we associate an implicit scheme of the form

$$
\text { (6) } \quad a^{2} \frac{u\left(x_{j+1}, t_{n}\right)-2 u\left(x_{j}, t_{n}\right)+u\left(x_{j-1}, t_{n}\right)}{h^{2}}-\frac{u\left(x_{j}, t_{n}\right)-u\left(x_{j}, t_{n-1}\right)}{k_{n}}=0 \text {. }
$$

Communicated by S. Matsuura March 17, 1973.

* Department of Applied Mathematics and Physics, Faculty of Engineering, Kyoto University, Kyoto. 
More precisely we shall introduce a family of rectangular lattices with space mesh $h$ and time steps $k_{n}(n=1,2, \ldots)$ where $h$ varies in such a way that $\frac{l}{h}=J$ is integer and $k_{n}$ 's are to be found so that the free boundary crosses lattices just at each mesh point $\left(x_{J+n}, t_{n}\right)$, where we put

$$
\begin{aligned}
& x_{j}=j h \quad(j=0, \pm 1, \pm 2, \ldots), \\
& t_{n}=\sum_{p=1}^{n} k_{p} \quad(n=1,2, \ldots) .
\end{aligned}
$$

With reference to given positive numbers $h$ and $k_{n}$ we introduce the divided differences

$$
\begin{aligned}
& u_{x}\left(x_{j}, t_{n}\right)=\frac{1}{h}\left[u\left(x_{j+1}, t_{n}\right)-u\left(x_{j}, t_{n}\right)\right], \\
& u_{\bar{x}}\left(x_{j}, t_{n}\right)=\frac{1}{h}\left[u\left(x_{j}, t_{n}\right)-u\left(x_{j-1}, t_{n}\right)\right], \\
& u_{x \bar{x}}\left(x_{j}, t_{n}\right)=\frac{1}{h^{2}}\left[u\left(x_{j+1}, t_{n}\right)-2 u\left(x_{j}, t_{n}\right)+u\left(x_{j-1}, t_{n}\right)\right], \\
& u_{\bar{t}}\left(x_{j}, t_{n}\right)=\frac{1}{k_{n}}\left[u\left(x_{j}, t_{n}\right)-u\left(x_{j}, t_{n-1}\right)\right] .
\end{aligned}
$$

Then $u=u\left(x_{j}, t_{n}\right)$ shall be the function defined for $\left(x_{j}, t_{n}\right)$ in the lattice which satisfies the recursion formula

$$
a^{2} u_{x \bar{x}}\left(x_{j}, t_{n}\right)-u_{\bar{t}}\left(x_{j}, t_{n}\right)=0
$$

and the boundary conditions

$$
\begin{aligned}
u\left(0, t_{n}\right) & =f\left(t_{n}\right), \\
u\left(y_{n}, t_{n}\right) & =0, \quad n=1,2, \ldots
\end{aligned}
$$

where $y_{n}=x_{J+n}$, and the initial condition

$$
u\left(x_{j}, 0\right)=\varphi\left(x_{j}\right), \quad j=0,1,2, \ldots, J .
$$

Our essential idea is that $k_{n}$ shall be determined from an analogue to the Stefan condition of the form 


$$
\frac{h}{k_{n}}=\kappa u_{\bar{x}}\left(y_{n}, t_{n}\right)+\beta \frac{k_{n}}{\sqrt{h}}, \quad n=1,2,3, \ldots
$$

with an artificial heat flow term $\beta \frac{k_{n}}{\sqrt{h}}$ ( $\beta$ is a suitable positive constant).

We can prove that for $h \rightarrow 0, u\left(x_{j}, t_{n}\right)$ and $y_{n}$ approach functions $u(x, t)$ and $y(t)$ under suitable conditions and this pair of functions $\{u(x, t), y(t)\}$ is a solution of (1)-(5).

We have now several works on difference schemes (Douglas and Gallie [1], Vasilev [2] etc). They treat the case in which an inhomogeneous Neumann type boundary condition is imposed at a fixed boundary and it is assumed that the inhomogeneous term is bounded away from zero. In that case a Stefan condition becomes equivalent to an integral relation which is effectively used in the iteration calculation. If the condition, for example,

$$
\frac{\partial u}{\partial x}(0, t)=1
$$

is imposed instead of (2), then the Stefan's condition (5) can be replaced by

$$
y(t)=l+\kappa t+\frac{\kappa}{a^{2}}\left(\int_{0}^{y(t)} u(x, t) d x-\int_{0}^{l} \varphi(x) d x\right)
$$

And we can consider the system (1), (12), (3), (4) and (5') instead of the system (1), (12), (3), (4) and (5), while in the case of Dirichlet type boundary condition as here considered by us such replacement cannot be done and in a case of homogeneous Neumann type boundary condition such replacement does not play an effective role. In our new scheme such restriction can be ridden. But essential restriction is that $l>0$ which may depend only on our method of proof.

Here we consider only the case of Dirichlet type boundary condition. The case of homogeneous Neumann type boundary condition can be treated in the same way. More general case including multi-phase problems also may be treated. 


\section{§1. Statement of the Main Result}

We shall essentially concerned with the problem (1)-(5): Find $u(x, t)$ and $y(t)>0$ such that

$$
\begin{aligned}
& \text { (1.1) } \quad a^{2} \frac{\partial^{2} u}{\partial x^{2}}-\frac{\partial u}{\partial t}=0 \quad(a \text { : const.) for } 0<x<y(t), t>0, \\
& (1.2) \quad u(0, t)=f(t) \quad \text { where } f(t) \leqq 0 \text { and } t>0, \\
& (1.3) \quad u(y(t), t)=0 \quad \text { for } t>0 \text { and } y(0)=l>0, \\
& \text { (1.4) } u(x, 0)=\varphi(x) \quad \text { where } \varphi(x) \leqq 0,0 \leqq x \leqq l, \text { and } \varphi(0)=f(0), \\
&
\end{aligned}
$$

$$
\dot{y}(t)=\kappa \frac{\partial u}{\partial x}(y(t), t) \quad \text { for } \quad t>0
$$

The assumptions $f \leqq 0, \varphi \leqq 0$ result from the physical background. Existence and uniqueness theorem about the last problem is well known. Furthermore it is known that under the assumption the function $x=y(t)$ is monotone nondecreasing in $t$ (Friedman [3]).

We consider the following difference analogue: Find $\left\{u\left(x_{j}, t_{n}\right)\right\}$ and positive $\left\{k_{n}\right\}$ such that

$$
\begin{array}{ll}
a^{2} u_{x \bar{x}}\left(x_{j}, t_{n}\right)-u_{\bar{t}}\left(x_{j}, t_{n}\right)=0, & \text { for } 0<x_{j}<y_{n}, t_{n}>0, \\
u\left(0, t_{n}\right)=f\left(t_{n}\right)(\leqq 0) & \text { for } t_{n}>0, \\
u\left(y_{n}, t_{n}\right)=0 & \text { for } t_{n}>0 \text { and } y_{0}=l>0, \\
u\left(x_{j}, 0\right)=\varphi\left(x_{j}\right)(\leqq 0) & \text { for } 0 \leqq x_{j} \leqq l \text { and } \varphi(l)=0, \\
\frac{h}{k_{n}}=\kappa v_{n}+\beta \frac{k_{n}}{\sqrt{h}} & \text { for } t_{n}>0,
\end{array}
$$

or

$$
\frac{h}{k_{n}}=\frac{1}{2}\left(\kappa v_{n}+\sqrt{\kappa^{2} v_{n}^{2}+4 \beta \sqrt{h}}\right),
$$


where

(1.11) $\quad y_{n}=x_{J+n}=(J+n) h, \quad J h=l$

(1.12) $t_{n}=\sum_{p=1}^{n} k_{p}$,

(1.13) $\quad v_{n}=u_{\bar{x}}\left(y_{n}, t_{n}\right)$.

Assume that we have already the solutions $u\left(x_{j}, t_{p}\right), k_{p}$ for $p \leqq n-1$.

Then we shall solve the difference scheme (2.6)-(2.8), (1.10) by the iteration process

$$
a^{2} u_{x \bar{x}}^{(s)}\left(x_{j}, t_{n}\right)-\frac{u^{(s)}\left(x_{j}, t_{n}\right)-u\left(x_{j}, t_{n-1}\right)}{k_{n}^{(s)}}=0, j=1,2, \ldots, J+n-1,
$$

(1.15) $\quad u^{(s)}\left(0, t_{n}\right)=f\left(t_{n}\right)$,

(1.16) $\quad u^{(s)}\left(y_{n}, t_{n}\right)=0$,

$$
k_{n}^{(s+1)}=\frac{\sqrt{h}}{2 \beta}\left[-\kappa v_{n}^{(s)}+\sqrt{\kappa^{2} v_{n}^{(s)^{2}}+4 \beta \sqrt{h}}\right], \quad s=1,2,3, \ldots,
$$

(1.18) $\quad k_{n}^{(1)}=k_{n-1}$.

We have

Theorem. Assume that $f(t) \in C^{1}(0<t<T), \varphi(x) \in C^{2}(0<x<l)$. Then

i) At each time step $t=t_{n}$, the iteration process (2.14)-(2.18) converges as $s \rightarrow \infty$ and the limits $\left\{u\left(x_{j}, t_{n}\right)\right\}, k_{n}$ satisfy the equations (2.6)-(2.10).

ii) The functions $\left\{u\left(x_{j}, t_{n}\right)\right\},\left\{y_{n}\right\}$ determined by (2.6)-(2.10) converge uniformly to the solution $u(x, t), y(t)$ of (2.1)-(2.5) respectively as $h \rightarrow \infty$.

We shall prove this theorem in $\S 3$ and $\S 4$. Before the proof we shall give some preliminaries in the next section. In $\S 5$ we shall give some numerical examples. 


\section{§2. Preliminaries}

Define for $r, j \geqq 0$,

$$
\begin{gathered}
g\left(x_{r}, \xi_{j} ; t_{n}, \tau_{p-1}\right)=\left\{\begin{array}{lc}
\frac{1}{2 \pi h} \int_{-\pi}^{\pi} \prod_{q=p}^{n} \Lambda_{q}^{-1}\left[e^{-i(r-j)_{\omega}}-e^{-i(r+j) \omega}\right] d \omega \\
\left.\frac{1}{h} \delta_{r, j} *\right) & \text { for } n \geqq p,
\end{array}\right. \\
G\left(x_{r}, \xi_{j} ; t_{n}, \tau_{p-1}\right)=\left\{\begin{array}{lc}
\frac{1}{2 \pi h} \int_{-\pi}^{\pi} \prod_{q=p}^{n} \Lambda_{q}^{-1}\left[e^{-i(r-j)_{\omega}}+e^{-i(r+j-1) \omega}\right] d \omega \\
\frac{1}{h} \delta_{r, j} & \text { for } n \geqq p,
\end{array}\right.
\end{gathered}
$$

where $\Lambda_{q}=1+4 \lambda_{q} \sin ^{2} \frac{\omega}{2}, \lambda_{q}=\frac{a^{2} k_{q}}{h^{2}}$.

Then these functions satisfy the equations

$$
\begin{array}{ll}
a^{2} g_{x \bar{x}}-g_{\bar{t}}=0, & a^{2} G_{x \bar{x}}-G_{\bar{t}}=0, \\
a^{2} g_{\xi \bar{\xi}}+g_{\tau}=0, & a^{2} G_{\xi \bar{\xi}}+G_{\tau}=0,
\end{array}
$$

and the boundary conditions

$$
\begin{aligned}
& g\left(0, \xi_{j} ; t_{n}, \tau_{p-1}\right)=g\left(x_{r}, 0 ; t_{n}, \tau_{p-1}\right)=0, \\
& G_{x}\left(0, \xi_{j} ; t_{n}, \tau_{p-1}\right)=G_{\xi}\left(x_{r}, 0 ; t_{n}, \tau_{p-1}\right)=0 .
\end{aligned}
$$

Furthermore we have the conjugate relations

$$
\begin{aligned}
& g_{\bar{x}}\left(x_{r}, \xi_{j} ; t_{n}, \tau_{p-1}\right)=-G_{\xi}\left(x_{r}, \xi_{j} ; t_{n}, \tau_{p-1}\right) \\
& G_{x}\left(x_{r}, \xi_{j} ; t_{n}, \tau_{p-1}\right)=-g_{\bar{\xi}}\left(x_{r}, \xi_{j} ; t_{n}, \tau_{p-1}\right) .
\end{aligned}
$$

We call $g$ the Green's function of the first boundary value problem in *) $\delta_{r, j}=\left\{\begin{array}{ll}1 & (r=j) \\ 0 & (r \neq j)\end{array} \quad\right.$ is Kronecker's delta. 
$x>0$ for the equation (2.6) and $G$ the Green's function of the second boundary value problem.

Lemma 1. Assume that $\left\{k_{n}\right\}$ are given. Then for the solution of the mixed initial-boundary value problem (1.6)-(1.9) we have

$$
\begin{aligned}
v_{n}= & {\left[1+a^{2} k_{n} G_{\xi}\left(y_{n}, \eta_{n} ; t_{n}, \tau_{n-1}\right)\right]^{-1} } \\
& \times\left[\sum_{j=1}^{J} h G\left(y_{n}, \xi_{j} ; t_{n}, 0\right) \varphi_{\bar{\xi}}\left(\xi_{j}\right)-\sum_{p=1}^{n} k_{p} G\left(y_{n}, 0 ; t_{n}, \tau_{p-1}\right) f_{\bar{\tau}}\left(\tau_{p}\right)\right. \\
& \left.-a^{2} \sum_{p=1}^{n-1} k_{p} G_{\xi}\left(y_{n}, \eta_{p} ; t_{n}, \tau_{p-1}\right) v_{p}\right]
\end{aligned}
$$

where $y_{n}=x_{J+n}, \quad \eta_{n}=\xi_{J+n}, \quad v_{n}=u_{\bar{x}}\left(y_{n}, t_{n}\right)$.

And we have also

$$
\begin{aligned}
v_{n}= & {\left[1+a^{2} k_{n} G_{\xi}\left(y_{n}, \eta_{n} ; t_{n}, \tau_{n-1}\right)\right]^{-1} } \\
& \times\left[-k_{n} G\left(y_{n}, 0 ; t_{n}, \tau_{n-1}\right) f_{\tau}\left(\tau_{n}\right)\right. \\
& \left.+\sum_{j=1}^{J+n-1} h G\left(y_{n}, \xi_{j} ; t_{n}, \tau_{n-1}\right) u_{\xi}\left(\xi_{j}, \tau_{n-1}\right)\right] .
\end{aligned}
$$

Proof. Assume that the functions $\varphi(\xi, \tau)$ and $\psi(\xi, \tau)$ satisfy

and

$$
\begin{aligned}
& a^{2} \varphi_{\xi \bar{\xi}}\left(\xi_{j}, \tau_{p}\right)-\varphi_{\tau}\left(\xi_{j}, \tau_{p}\right)=0 \\
& a^{2} \psi_{\xi \bar{\xi}}\left(\xi_{j}, \tau_{p-1}\right)+\psi r_{\tau}\left(\xi_{j}, \tau_{p-1}\right)=0 .
\end{aligned}
$$

Multiply the former by $h k_{p} \psi\left(\xi_{j}, \tau_{p}\right)$ and the latter by $h k_{p} \varphi\left(\xi_{j}, \tau_{p}\right)$, add each resulted equation over $j=1,2, \ldots, J+n-1$ and $p=1,2, \ldots, n$ and subtract the latter sum from the former. Then we have

$$
\begin{aligned}
& a^{2} \sum_{p=1}^{n} k_{p} \sum_{j=1}^{J+p-1} h\left[\psi\left(\xi_{j}, \tau_{p-1}\right) \varphi_{\xi \bar{\xi}}\left(\xi_{j}, \tau_{p}\right)-\varphi\left(\xi_{j}, \tau_{p}\right) \psi_{\xi \bar{\xi}}\left(\xi_{j}, \tau_{p-1}\right)\right] \\
& -\sum_{p=1}^{n} k_{p} \sum_{j=1}^{J+p-1} h\left[\varphi_{\tau}\left(\xi_{j}, \tau_{p-1}\right) \psi\left(\xi_{j}, \tau_{p-1}\right)+\varphi\left(\xi_{j}, \tau_{p}\right) \psi_{\bar{\tau}}\left(\xi_{j}, \tau_{p}\right)\right]=0 .
\end{aligned}
$$

Applying summation by parts,

$$
\sum_{j=1}^{J+n} h \psi\left(\xi_{j}, \tau_{n}\right) \varphi\left(\xi_{j}, \tau_{n}\right)=\sum_{j=1}^{J} h \psi\left(\xi_{j}, 0\right) \varphi\left(\xi_{j}, 0\right)+
$$




$$
\begin{aligned}
& +\sum_{p=1}^{n} h \psi\left(\eta_{p}, \tau_{p}\right) \varphi\left(\eta_{p}, \tau_{p}\right) \\
& +a^{2} \sum_{p=1}^{n} k_{p}\left[\psi\left(\eta_{p-1}, \tau_{p-1}\right) \varphi_{\xi}\left(\eta_{p-1}, \tau_{p}\right)-\psi_{\xi}\left(\eta_{p-1}, \tau_{p-1}\right) \varphi\left(\eta_{p-1}, \tau_{p}\right)\right] \\
& -a^{2} \sum_{p=1}^{n} k_{p}\left[\psi\left(0, \tau_{p-1}\right) \varphi_{\xi}\left(0, \tau_{p}\right)-\psi_{\xi}\left(0, \tau_{p-1}\right) \varphi\left(0, \tau_{p}\right)\right] .
\end{aligned}
$$

Now we take

$$
\varphi\left(\xi_{j}, \tau_{p}\right)=u\left(\xi_{j}, \tau_{p}\right), \quad \psi\left(\xi_{j}, \tau_{p}\right)=g\left(x_{r}, \xi_{j} ; t_{n}, \tau_{p}\right) .
$$

Then from (1.7), (1.8), (1.9), (2.4) and the equality

$$
u\left(\eta_{p-1}, \tau_{p}\right)=-h v_{p} \quad(\text { by }(1.8))
$$

we have

$$
\begin{aligned}
u\left(x_{r}, t_{n}\right)= & \sum_{j=1}^{J} h g\left(x_{r}, \xi_{j} ; t_{n}, 0\right) \varphi\left(\xi_{j}\right) \\
& +a^{2} \sum_{p=1}^{n} k_{p} g\left(x_{r}, \eta_{p} ; t_{n}, \tau_{p-1}\right) v_{p} \\
& +a^{2} \sum_{p=1}^{n} k_{p} g_{\xi}\left(x_{r}, 0 ; t_{n}, \tau_{p-1}\right) f\left(\tau_{p}\right) .
\end{aligned}
$$

Hence by (2.5)

$$
\begin{aligned}
u_{\bar{x}}\left(x_{r}, t_{n}\right)= & -\sum_{j=1}^{J} h G_{\xi}\left(x_{r}, \xi_{j} ; t_{n}, 0\right) \varphi\left(\xi_{j}\right) \\
& -a^{2} \sum_{p=1}^{n} k_{p} G_{\xi}\left(x_{r}, \eta_{p} ; t_{n}, \tau_{p-1}\right) v_{p} \\
& -a^{2} \sum_{p=1}^{n} k_{p} G_{\xi \bar{\xi}}\left(x_{r}, \xi_{1} ; t_{n}, \tau_{p-1}\right) f\left(\tau_{p}\right) .
\end{aligned}
$$

Using the equation (2.3) and applying summation by parts to (2.10) we have

$$
\begin{aligned}
u_{\bar{x}}\left(x_{r}, t_{n}\right)= & \sum_{j=1}^{J} h G\left(x_{r}, \xi_{j} ; t_{n}, 0\right) \varphi_{\bar{\xi}}\left(\xi_{j}\right)-\sum_{p=1}^{n} k_{p} G\left(x_{r}, 0 ; t_{n}, \tau_{p-1}\right) f_{r}\left(\tau_{p}\right) \\
& -a^{2} \sum_{p=1}^{n} k_{p} G_{\xi}\left(x_{r}, \eta_{p} ; t_{n}, \tau_{p-1}\right) v_{p} \\
& +G\left(x_{r}, \xi_{1} ; t_{n}, \tau_{n}\right) f\left(\tau_{n}\right) .
\end{aligned}
$$


In the last equation we take $r=J+n$ and solve this equation regarding $u_{\bar{x}}\left(y_{n}, t_{n}\right)=v_{n}$ as unknown. Then we get (2.6) since $G\left(y_{n}, \xi_{1} ; t_{n}, \tau_{n}\right)=0$.

In the same way we also have (2.7).

Lemma 2. For small $h$,

$$
1+a^{2} G_{\xi}\left(y_{n}, \eta_{n} ; t_{n}, \tau_{n-1}\right)>\frac{1}{4}
$$

Proof.

$$
\begin{aligned}
& 1+a^{2} G_{\xi}\left(y_{n}, \eta_{n} ; t_{n}, \tau_{n-1}\right) \\
& =1-\frac{\lambda_{n}}{\pi} \int_{-\pi}^{\pi} \Lambda_{n}^{-1} \sin ^{2} \frac{\omega}{2} d \omega-\frac{\lambda_{n}}{\pi} \int_{-\pi}^{\pi} \Lambda_{n}^{-1} \sin \{2(J+n)-1\} \omega \cdot \sin \frac{\omega}{2} d \omega \\
& >\frac{1}{2}\left(1+\frac{1}{\sqrt{1+4 \lambda_{n}}}\right)-\frac{\lambda_{n}}{\pi\left\{2(J+n)-\frac{1}{2}\right\}} \int_{-\pi}^{\pi}\left(\Lambda_{n}^{-2}+\frac{1}{2} \Lambda_{n}^{-1}\right) d \omega \\
& =\frac{1}{2}\left(1+\frac{1}{\sqrt{1+4 \lambda_{n}}}\right)-\frac{\lambda_{n}}{\pi\left\{2(J+n)-\frac{1}{2}\right\}}\left[\frac{1}{1+4 \lambda_{n}}+\frac{1}{\sqrt{\lambda_{n}}} \tan ^{-1} \sqrt{4 \lambda_{n}}\right]
\end{aligned}
$$

Since

$$
\left\{2(J+n)-\frac{1}{2}\right\} h>2 J h=2 l
$$

we have as $h, k_{n} \rightarrow 0$

$$
\begin{aligned}
1+a^{2} G_{\xi}\left(y_{n}, \eta_{n} ; t_{n}, \tau_{n-1}\right) & >\frac{1}{2}\left(1+\frac{1}{\sqrt{1+4 \lambda_{n}}}\right)-0(h)-0\left(\sqrt{k_{n}}\right) \\
& >\frac{1}{4} .
\end{aligned}
$$

\section{Lemma 3.}

$$
\left|G\left(y_{n}, 0 ; t_{n}, \tau_{p-1}\right)\right|<\frac{1}{a \sqrt{t_{n}-\tau_{p-1}}}
$$


Proof.

$$
\begin{aligned}
& \left|G\left(y_{n}, 0 ; t_{n}, \tau_{p-1}\right)\right|=\left|\frac{1}{2 \pi h} \int_{-\pi}^{\pi} \prod_{q=p}^{n} \Lambda_{q}^{-1}\left(e^{-i(J+n)_{\omega}}+e^{-i(J+n-1) \omega}\right) d \omega\right| \\
& \leqq \frac{1}{\pi h} \int_{-\pi}^{\pi}\left(1+4 \sum_{q=p}^{n} \lambda_{q} \sin ^{2} \frac{\omega}{2}\right)^{-1} d \omega=\frac{2}{h \sqrt{1+4 \sum_{q=p}^{n} \lambda_{q}}} \\
& <\frac{1}{a \sqrt{t_{n}-\tau_{p-1}}} .
\end{aligned}
$$

Lemma 4. For any function $\psi\left(\xi_{i}\right)$ we have

$$
\left|\sum_{j=1}^{J+p} h G\left(y_{n}, \xi_{j} ; t_{n}, \tau_{p}\right) \psi\left(\xi_{j}\right)\right|<\max _{j=1,2, \ldots, J+p}\left|\psi\left(\xi_{j}\right)\right|
$$

Proof. The function

$$
w\left(x_{r}, t_{n}\right)=\sum_{j=1}^{J+p} h G\left(x_{r}, \xi_{j} ; t_{n}, \tau_{p}\right) \psi\left(\xi_{j}\right)
$$

satisfies the difference equation (7) for $n>p,-\infty<j<\infty$ and has the Cauchy data

$$
w\left(x_{j}, t_{p}\right)= \begin{cases}\psi\left(x_{j}\right), & j=1,2, \ldots, J+p \\ \psi\left(x_{-j+1}\right), & j=0,1,2, \ldots,(J+p)+1 \\ 0, & \text { otherwise. }\end{cases}
$$

Thus (2.14) follows from the well-known maximum principle.

Lemma 5. For $p<n$, we have

$$
\frac{1}{\pi h^{2}} \int_{-\pi}^{\pi} \prod_{q=p}^{n} \Lambda_{q}^{-1} \sin ^{2} \frac{\omega}{2} d \omega \leqq \frac{h}{2 \sqrt{2} a^{3}\left(t_{n}-\tau_{p-1}-\hat{k}\right)^{\frac{3}{2}}}
$$

where

$$
\hat{k}=\hat{k}(p, n)=\max _{q=p, \ldots, n} k_{q}
$$

In particular we obtain for $p=n-1$ that 


$$
\frac{1}{\pi h^{2}} \int_{-\pi}^{\pi} \Lambda_{n-1}^{-1} \Lambda_{n}^{-1} \sin ^{2} \frac{\omega}{2} d \omega<\frac{h}{4 a^{3} \sqrt{k_{n-1} k_{n}}\left(\sqrt{k_{n-1}}+\sqrt{k_{n}}\right)} .
$$

Proof. (2.17) can be obtained by elementary integration. We shall prove (2.15). First we shall show that there are two partial sums $\sum_{\mathrm{I}} k_{q}$ and $\sum_{\text {II }} k_{r}(q \neq r)$ such that

$$
t_{n}-\tau_{p-1}=\sum_{\mathrm{I}} k_{q}+\sum_{\mathrm{II}} k_{r}
$$

and

$$
\sum_{\mathrm{I}} k_{q} \text { and } \sum_{\mathrm{II}} k_{r}>\frac{1}{2}\left(t_{n}-\tau_{p-1}-\hat{k}\right)
$$

hold. In fact it is clear that we can select two partial sums $\sum^{\prime} k_{q}, \Sigma^{\prime \prime} k_{r}$ such that

$$
\begin{aligned}
& \sum_{q=p}^{n} k_{q}=\Sigma^{\prime} k_{q}+\Sigma^{\prime \prime} k_{r}+\hat{k} \quad\left(q \neq r, k_{q} \neq \hat{k}, k_{r} \neq \hat{k}\right), \\
& \sum^{\prime} k_{q} \leqq \Sigma^{\prime \prime} k_{r} \leqq \frac{1}{2} \sum_{q=p}^{n} k_{q} .
\end{aligned}
$$

Hence

$$
\begin{aligned}
& \sum^{\prime \prime} k_{r} \geqq \frac{1}{2}\left(\sum_{q=p}^{n} k_{q}-\hat{k}\right), \\
& \sum^{\prime} k_{q}+\hat{k} \geqq \frac{1}{2} \sum_{q=p}^{n} k_{q} .
\end{aligned}
$$

Let

$$
\sum_{\mathrm{I}} k_{q}=\Sigma^{\prime} k_{q}+\hat{k}, \quad \sum_{\mathrm{II}} k_{r}=\Sigma^{\prime \prime} k_{r},
$$

which then satisfy (2.18) and (2.19).

It follows from (2.19) that

$$
\frac{1}{\pi h^{2}} \int_{-\pi}^{\pi} \prod_{q=p}^{n} \Lambda_{q}^{-1} \sin ^{2} \frac{\omega}{2} d \omega<\frac{1}{\pi h^{2}} \int_{-\pi}^{\pi} \frac{\sin ^{2} \frac{\omega}{2} d \omega}{\left(1+4 \sum_{\mathrm{I}} \lambda_{q} \sin ^{2} \frac{\omega}{2}\right)\left(1+4 \sum_{\mathrm{II}} \lambda_{r} \sin ^{2} \frac{\omega}{2}\right)}
$$




$$
<\frac{1}{\pi h^{2}} \int_{-\pi}^{\pi} \frac{\sin ^{2} \frac{\omega}{2} d \omega}{\left\{1+\frac{2 a^{2}}{h^{2}}\left(t_{n}-\tau_{p-1}-\hat{k}\right) \sin ^{2} \frac{\omega}{2}\right\}^{2}}
$$

and by elementary integration*)

$$
=\frac{1}{h^{2}\left[1+\frac{2 a^{2}}{h^{2}}\left(t_{n}-\tau_{p-1}-\hat{k}\right)\right]^{\frac{3}{2}}}<\frac{h}{2 \sqrt{2} a^{3}\left(t_{n}-\tau_{p-1}-\hat{k}\right)^{\frac{3}{2}}} .
$$

This proves (2.15).

Denote by $V_{h}$ a bound for $\frac{h}{k_{p}}(p=1,2, \ldots, n)$ :

$$
\frac{h}{k_{p}}<V_{h}
$$

Then we have

$$
\begin{aligned}
& (n-p+1) h<\left(t_{n}-\tau_{p-1}\right) V_{h}, \\
& (n-p) h<\left(t_{n}-\tau_{p-1}-\hat{k}\right) V_{h} .
\end{aligned}
$$

Lemma 6. If $\gamma=1$ ( $K$ is arbitrary), or $\gamma=\frac{1}{2}$ and $K$ is a half-integer, then

$$
\left|I_{K}\right| \equiv\left|\frac{1}{2 \pi h^{2}} \int_{-\pi}^{\pi} \prod_{q=p}^{n} \Lambda_{q}^{-1} \sin \gamma \omega \cdot \sin K \omega d \omega\right|
$$

$$
<\frac{(1+2 \sqrt{2}) \gamma}{2 a} \cdot \frac{1}{K h \sqrt{t_{n}-\tau_{p-1}-\hat{k}}}+\frac{\sqrt{2} \gamma}{a} \cdot \frac{\hat{k}}{K h\left(t_{n}-\tau_{p-1}-\hat{k}\right)^{3 / 2}}
$$

or alternatively

$$
\left|I_{K}\right|<\frac{3 \gamma}{2 a} \frac{\sqrt{t_{n}-\tau_{p-1}}}{K h^{2}} V_{h}
$$

$$
\int_{-\pi}^{\pi} \frac{\sin ^{2} \frac{\omega}{2}}{\left(1+\alpha \sin ^{2} \frac{\omega}{2}\right)^{2}} d \omega=\frac{\pi}{(1+\alpha)^{3 / 2}} \quad(\alpha: \text { const. })
$$


Proof. We put

$$
\prod_{q=p}^{n} \Lambda_{q}^{-1} \sin \gamma \omega=\psi(\omega)
$$

Applying integration by parts to $I_{K}$ we have

$$
\left|I_{K}\right|=\frac{1}{2 K h^{2} \pi}\left|\int_{-\pi}^{\pi} \psi^{\prime}(\omega) \cos K \omega d \omega\right|
$$

Since

$$
\text { (2.26) } \quad \psi^{\prime}(\omega)=\gamma \prod_{q=p}^{n} \Lambda_{q}^{-1}\left[\cos \gamma \omega-\sum_{q=p}^{n} 8 \Lambda_{q}^{-1} \lambda_{q} \sin ^{2} \frac{\omega}{2} \cos ^{2 \gamma} \frac{\omega}{2}\right]
$$

it follows that

$$
\begin{aligned}
\left|I_{K}\right|<\frac{\gamma}{2 K h^{2} \pi}\left[\int_{-\pi}^{\pi} \frac{d \omega}{1+4 \sum_{q=p}^{n} \lambda_{q} \sin ^{2} \frac{\omega}{2}} *\right) \\
\left.\quad+8 \sum_{q=p}^{n} \lambda_{q} \int_{-\pi}^{\pi} \prod_{q=p}^{n} \Lambda_{q}^{-1} \sin ^{2} \frac{\omega}{2} d \omega\right]
\end{aligned}
$$

or alternatively

$$
\left|I_{K}\right|<\frac{\left(n-p+\frac{3}{2}\right) \gamma}{K h^{2} \pi} \int_{-\pi}^{\pi} \frac{d \omega}{1+4 \sum_{q=p}^{n} \lambda_{q} \sin ^{2} \frac{\omega}{2}}
$$

and further that from Lemma 5,

$$
\begin{aligned}
\left|I_{K}\right| & <\frac{\gamma}{2 a} \frac{1}{K h \sqrt{t_{n}-\tau_{p-1}}}+\frac{\sqrt{2} \gamma}{a^{3}} \frac{t_{n}-\tau_{p-1}}{K h\left(t_{n}-\tau_{p-1}-\hat{k}\right)} \\
& =\frac{(1+2 \sqrt{2}) \gamma}{2 a} \frac{1}{K h \sqrt{t_{n}-\tau_{p-1}}}+\frac{\sqrt{2} \gamma}{a} \frac{\hat{k}}{K h\left(t_{n}-\tau_{p-1}-\hat{k}\right)^{3 / 2}},
\end{aligned}
$$

or alternatively

$$
\int_{-\pi}^{\pi} \frac{d \omega}{1+4 \lambda \sin ^{2} \frac{\omega}{2}}=\frac{2 \pi}{\sqrt{1+4 \lambda}}<\frac{\pi}{\sqrt{\lambda}}
$$




$$
\left|I_{K}\right|<\frac{\left(n-p+\frac{3}{2}\right) h \gamma}{K h^{2} a \sqrt{t_{n}-\tau_{p-1}}}<\frac{3 \gamma}{2 a} \frac{\sqrt{t_{n}-\tau_{p-1}}}{K h^{2}} V_{h} .
$$

\section{Lemma 7.}

$$
\begin{aligned}
& \left|G_{\xi}\left(y_{n}, \eta_{p} ; t_{n}, \tau_{p-1}\right)\right| \\
& <\left[\frac{2+\pi}{2 \sqrt{2} a^{3}} V_{h}+\frac{1+2 \sqrt{2}}{2 a} \cdot \frac{1}{y_{n}+\eta_{p}}\right] \frac{1}{\sqrt{t_{n}-\tau_{p-1}-\hat{k}}} \\
& \quad+\frac{\sqrt{2}}{a\left(y_{n}+\eta_{p}\right)} \cdot \frac{\hat{k}}{\sqrt{t_{n}-\tau_{p-1}-\hat{k}}}
\end{aligned}
$$

or alternatively

$$
\left|G_{\xi}\left(y_{n}, \eta_{p} ; t_{n}, \tau_{p-1}\right)\right|<\frac{2+\pi}{2 \sqrt{2} a^{3}} \frac{V_{h}}{\sqrt{t_{n}-\tau_{p-1}-\hat{k}}}
$$

$$
+\frac{3 \sqrt{t_{n}-\tau_{p-1}}}{2 a\left(y_{n}+\eta_{p}\right) h} V_{h}
$$

Proof. It follows from the definition that

$$
\begin{aligned}
& G_{\xi}\left(y_{n}, \eta_{p}: t_{n}, \tau_{p-1}\right) \\
& =\frac{1}{\pi h^{2}} \int_{-\pi}^{\pi} \prod_{q=p}^{n} \Lambda_{q}^{-1}\left[-2 \sin ^{2} \frac{\omega}{2} \sin (J+n) \omega \sin (J+p) \omega\right. \\
& \left.\quad+\sin \frac{\omega}{2} \sin (n-p) \omega \cos \frac{\omega}{2}\right] d \omega-I_{2 J+n+p} \quad \text { (see (2.23)). }
\end{aligned}
$$

Hence by Lemma $5,(2.22)$ and $6 *$ )

$$
\begin{aligned}
& \left|G_{\xi}\left(y_{n}, \eta_{p} ; t_{n}, \tau_{p-1}\right)\right| \\
& <\frac{[2+(n-p) \pi]}{\pi h^{2}} \int_{-\pi}^{\pi} \prod_{q=p}^{n} A_{q}^{-1} \sin ^{2} \frac{\omega}{2} d \omega+\left|I_{2 J+n+p}\right| \\
& \left.<\frac{2+\pi}{2 \sqrt{2} a^{3}} \frac{(n-p) h}{\left(t_{n}-\tau_{p-1}-\hat{k}\right)^{3 / 2}}+\left|I_{2 J+n+p}\right| * *\right)
\end{aligned}
$$

*) $|\sin (n-p) \omega|<(n-p)|\omega|<(n-p) \pi\left|\sin \frac{\omega}{2}\right|, \quad$ for $\quad|\omega|<\pi$

**) $2+(n-p) \pi<(2+\pi)(n-p)$ 


$$
\begin{gathered}
<\left[\frac{2+\pi}{2 \sqrt{2 a^{3}}} V_{h}+\frac{1+2 \sqrt{2}}{2 a} \frac{1}{y_{n}+\eta_{p}}\right] \frac{1}{\sqrt{t_{n}-\tau_{p-1}-\hat{k}}} \\
+\frac{\sqrt{2}}{a} \frac{\hat{k}}{\left(y_{n}+\eta_{p}\right)\left(t_{n}-\tau_{p-1}-\hat{k}\right)^{3 / 2}}
\end{gathered}
$$

or alternately

$$
<\frac{2+\pi}{2 \sqrt{2} a^{3}} \cdot \frac{V_{h}}{\sqrt{t_{n}-\tau_{p-1}-\hat{k}}}+\frac{3}{2 a} \frac{\sqrt{t_{n}-\tau_{p-1}}}{\left(y_{n}+\eta_{p}\right) h} V_{h} .
$$

Lemma 8. Assume that $k_{1} \geqq k_{2}$. If $h$ is small with $\frac{h}{k_{i}}<$ const. $(i=1,2)$, we have

$$
\left|\Phi_{K}\right| \equiv\left|\frac{1}{2 \pi h^{2}} \int_{-\pi}^{\pi} \Lambda_{1}^{-1} \Lambda_{2}^{-1} \sin \omega \sin K \omega d \omega\right|<\frac{\pi}{4 a^{3}} \frac{K h}{k_{1} \sqrt{k_{2}}}
$$

or for $K h>3 a \sqrt{k_{1}}$, or alternately

$$
<\frac{K h}{4 a^{3} k_{1}{ }^{3 / 2}} e^{-\frac{K h}{a \sqrt{k_{i}}}}+\frac{\pi^{3}}{4 a^{3}} \frac{h}{k_{1} \sqrt{k_{2}}} .
$$

Proof. Since

$$
\left|\Phi_{K}\right|<\frac{K}{h^{2}} \int_{-\pi}^{\pi} \Lambda_{1}^{1} \Lambda_{2}^{-1} \sin ^{2} \frac{\omega}{2} d \omega
$$

we get (2.31) by (2.17) in Lemma 5 .

Next we put

$$
\Phi_{K}=\Phi_{K 1}+\Phi_{K 2}+\Phi_{K 3}
$$

where

$$
\begin{aligned}
& \Phi_{K 1}=\frac{1}{2 \pi h^{2}} \int_{-\pi}^{\pi} \frac{\omega \sin K \omega}{\left(1+\lambda_{1} \omega^{2}\right)\left(1+\lambda_{2} \omega^{2}\right)} d \omega, \\
& \Phi_{K 2}=\frac{1}{2 \pi h^{2}} \int_{-\pi}^{\pi} \frac{(\sin \omega-\omega) \sin K \omega}{\Lambda_{1} \Lambda_{2}} d \omega
\end{aligned}
$$

and

$$
\Phi_{K 3}=\frac{1}{2 \pi h^{2}} \int_{-\pi}^{\pi}\left[\frac{1}{\Lambda_{1} \Lambda_{2}}-\frac{1}{\left(1+\lambda_{1} \omega^{2}\right)\left(1+\lambda_{2} \omega^{2}\right)}\right] \omega \sin K \omega d \omega
$$


Put $\omega=h \xi$. Then

$$
\begin{aligned}
\left|\Phi_{K 1}\right|< & \frac{1}{\pi}\left|\int_{0}^{\infty} \frac{\xi \sin K h \xi}{\left(1+\lambda_{1} h^{2} \xi^{2}\right)\left(1+\lambda_{2} h^{2} \xi^{2}\right)} d \xi\right|^{*)} \\
& +\frac{1}{\pi} \int_{\frac{\pi}{h}}^{\infty} \frac{\xi d \xi}{\left(1+\lambda_{1} h^{2} \xi^{2}\right)\left(1+\lambda_{2} h^{2} \xi^{2}\right)} \\
= & \frac{\exp \left(-\frac{K h}{a \sqrt{k_{1}}}\right)-\exp \left(-\frac{K h}{a \sqrt{k_{2}}}\right)}{2 a^{2}\left(k_{1}-k_{2}\right)}+\frac{1}{2 \pi a^{2}\left(k_{1}-k_{2}\right)} \log \frac{\lambda_{2}^{-1}+\pi^{2}}{\lambda_{1}^{-1}+\pi^{2}} .
\end{aligned}
$$

Using monotonicity of the function $\frac{K h}{k^{3 / 2}} \exp \left(-\frac{K h}{a \sqrt{k}}\right)$ with respect to $k$ for $K h>3 a \sqrt{k}$ we conclude that

$$
\left|\Phi_{K 1}\right|<\frac{K h}{4 a^{3} k_{1}^{3 / 2}} \exp \left(-\frac{K h}{a \sqrt{k_{1}}}\right)+\frac{h^{2}}{2 \pi^{3} a^{2} k_{2}^{2}}
$$

Next by (2.17) in Lemma 5 ,

$$
\begin{aligned}
\left|\Phi_{K 2}\right| & <\frac{\pi^{2}}{3 h^{2}} \int_{0}^{\pi} \Lambda_{1}^{-1} \Lambda_{2}^{-1} \sin ^{2} \frac{\omega}{2} d \omega<\frac{\pi^{3}}{12 a^{3}} \frac{h}{\sqrt{k_{1} k_{2}}\left(\sqrt{k_{1}}+\sqrt{k_{2}}\right)} \\
& <* \frac{\pi^{3} h}{12 a^{3} k_{1} \sqrt{k_{2}}}
\end{aligned}
$$

and

$$
\left|\Phi_{K 3}\right|<\frac{1}{\pi h^{2}} \int_{0}^{\pi} \frac{\left(\lambda_{1}+\lambda_{2}\right)\left(\omega^{2}-\sin ^{2} \frac{\omega}{2}\right)+\lambda_{1} \lambda_{2}\left(\omega^{4}-16 \sin ^{4} \frac{\omega}{2}\right)}{\Lambda_{1} \Lambda_{2}\left(1+\lambda_{1} \omega^{2}\right)\left(1+\lambda_{2} \omega^{2}\right)} d \omega
$$

*)

$\int_{0}^{\infty} \frac{\xi \sin m \xi}{\left(1+a^{2} \xi^{2}\right)\left(1+b^{2} \xi^{2}\right)} d \xi= \begin{cases}\frac{\pi}{2\left(a^{2}-b^{2}\right)}\left(e^{-\frac{m}{a}}-e^{-\frac{m}{b}}\right) & (a \neq b) \\ \frac{m \pi}{4 a^{3}} e^{-\frac{m}{a}} & (a=b)\end{cases}$

**) $\quad|\sin \omega-\omega|<\frac{1}{3}\left|\omega^{3}\right|<\frac{\pi^{3}}{3} \sin ^{2} \frac{\omega}{2} \quad$ for $|\omega| \leqq \pi$

***) $\omega^{2}-4 \sin ^{2} \frac{\omega}{2}<\frac{1}{6} \omega^{4}$,

$\omega^{4}-16 \sin ^{4} \frac{\omega}{2}<\frac{1}{3} \omega^{6}$ 


$$
\begin{aligned}
& \leqq \frac{\left(\lambda_{1}+\lambda_{2}\right)}{6 \pi h^{2} \lambda_{1} \lambda_{2}} \int_{0}^{\infty} \frac{\omega d \omega}{\left(1+\frac{4}{\pi^{2}} \lambda_{2} \omega^{2}\right)^{2}}+\frac{\pi^{2}}{12 h^{2} \lambda_{1}} \int_{0}^{\pi} \frac{d \omega}{1+4 \lambda_{2} \sin ^{2} \frac{\omega}{2}} \\
& <\frac{\pi h^{2}}{16 a^{4} k_{2}^{2}}+\frac{\pi^{3} h}{24 a^{3} k_{1} \sqrt{k_{2}}} .
\end{aligned}
$$

From (2.33)-(2.36) we get

$$
\left|\Phi_{K}\right|<\frac{K h}{4 a^{3} k_{1}^{3 / 2}} \exp \left(-\frac{K h}{a \sqrt{k_{1}}}\right)+\left(\frac{1}{2 \pi^{3} a^{2}}+\frac{\pi}{16 a^{4}}\right) \frac{h^{2}}{k_{2}^{2}}+\frac{\pi^{3}}{8 a^{3}} \frac{h}{k_{1} \sqrt{k_{2}}}
$$

and for small $h$ with $\frac{h}{k_{i}}<$ const. $\quad(i=1,2)$,

$$
\left|\Phi_{K}\right|<\frac{K h}{4 a^{3} k_{1}^{3 / 2}} \exp \left(-\frac{K h}{a \sqrt{k_{1}}}\right)+\frac{\pi^{3}}{4 a^{3}} \cdot \frac{h}{k_{1} \sqrt{k_{2}}} .
$$

This proves the second part of Lemma 8 .

\section{§3. Convergence of the Iteration Procedure}

We shall prove the first part of Theorem. It is supposed that we know already $u\left(x_{j}, t_{n-1}\right)(j=0,1,2, \ldots, J+n-1)$ and $k_{n-1}$ and have the estimates

$$
\begin{aligned}
& \max _{j}\left|u_{\bar{x}}\left(x_{j}, t_{n-1}\right)\right|<\bar{M}, \\
& \max _{j}\left|u_{x \bar{x}}\left(x_{j}, t_{n-1}\right)\right|<\frac{\bar{M}}{a^{2}} .
\end{aligned}
$$

In order to prove convergence of the iteration procedure (1.14)-(1.18) it is sufficient to show that there is a constant $\delta(0<\delta<1)$ such that

$$
\left|v_{n}^{(s)}-v_{n}^{(s-1)}\right|<\delta\left|v_{n}^{(s-1)}-v_{n}^{(s-2)}\right| \quad(s=3,4, \ldots) .
$$

In fact it follows from (3.2) that $v_{n}^{(s)}$ converges as $s \rightarrow \infty$ and hence $k_{n}^{(s)}$ also converges to a limit $k_{n}$. From maximum principle $u^{(s)}\left(x_{j}, t_{n}\right)$ are uniformly bounded and hence each subsequence $u^{\left(s_{i}\right)}\left(x_{j}, t_{n}\right)$ converges to each limit $u\left(x_{j}, t_{n}\right)(j=0,1,2, \ldots, J+n)$. It is clear that the limit function satisfies the equation 


$$
a^{2} u_{x \bar{x}}\left(x_{j}, t_{n}\right)-\frac{u\left(x_{j}, t_{n}\right)-u\left(x_{j}, t_{n-1}\right)}{k_{n}}=0
$$

and the conditions $u\left(0, t_{n}\right)=f\left(t_{n}\right), u\left(y_{n}, t_{n}\right)=0$. Since such function is uniquely determined, the sequence $u^{(s)}\left(x_{j}, t_{n}\right)$ itself converges to the same limits $u\left(x_{j}, t_{n}\right),(j=0,1, \ldots, J+n)$.

Now we shall show that (3.2) is valid under some conditions. Applying the formula (2.7) in Lemma 1 to the solution of (1.14)-(1.16) we have

$$
\begin{aligned}
v_{n}^{(s)}= & {\left[1+a^{2} k_{n}^{(s)} G_{\xi}\left(y_{n}, \eta_{n} ; t_{n-1}+k_{n}^{(s)}, \tau_{n-1}\right)\right]^{-1} } \\
& \times\left[\sum_{j=1}^{J+n-1} h G\left(y_{n}, \xi_{j} ; t_{n-1}+k_{n}^{(s)}, \tau_{n-1}\right) u_{\xi}\left(\xi_{j}, \tau_{n-1}\right)\right. \\
& \left.-G\left(y_{n}, 0 ; t_{n-1}+k_{n}^{(s)}, \tau_{n-1}\right)\left\{f\left(\tau_{n-1}+k_{n}^{(s)}\right)-f\left(\tau_{n-1}\right)\right\}\right] .
\end{aligned}
$$

First we get from Lemmas 2, 3, 4

$$
\left|v_{n}^{(s)}\right|<4\left(\tilde{M}+\frac{\sqrt{k_{n}^{(s)}}}{a} M_{1}\right)
$$

Since $k_{n}^{(s)}$ is at most $\frac{h^{3 / 4}}{\sqrt{\beta}}$ from (1.17), we have for small $h$

$$
\left|v_{n}^{(s)}\right|<4 \bar{M}+1=M, \quad s=1,2,3, \ldots
$$

Hence it follows from (1.17) that

$$
\begin{aligned}
\frac{h}{k_{n}^{(s)}} & =\frac{1}{2}\left(\sqrt{\kappa^{2} v_{n}^{(s-1)^{2}}+4 \beta \sqrt{h}}+\kappa v_{n}^{(s-1)}\right) \\
& <\kappa M+\frac{\beta}{\kappa M} \sqrt{h} \\
& <2 \kappa M
\end{aligned}
$$

for small $h$.

We consider the difference $v_{n}^{(s)}-v_{n}^{(s-1)}$ : using the notation

$$
D\left(\cdot\left(k_{n}^{(s)}\right)\right)=\cdot\left(k_{n}^{(s)}\right)-\cdot\left(k_{n}^{(s-1)}\right)
$$

we have 


$$
\begin{gathered}
\left|v_{n}^{(s)}-v_{n}^{(s-1)}\right|<\left|D\left(\Gamma_{1}^{-1}\left(k_{n}^{(s)}\right)\right)\right| \cdot\left|\Gamma_{2}\left(k_{n}^{(s)}\right)\right| \\
+\left|\Gamma_{1}^{-1}\left(k_{n}^{(s-1)}\right)\right| \cdot\left[\mid \sum_{j=1}^{J+n-1} h D\left(G\left(y_{n}, \xi_{j} ; t_{n-1}+k_{n}^{(s)}, \tau_{n-1}\right) u_{\bar{\xi}}\left(\xi_{j}, \tau_{n-1}\right) \mid\right.\right.
\end{gathered}
$$

$$
\begin{aligned}
& +\left|G\left(y_{n}, 0 ; t_{n-1}+k_{n}^{(s)}, \tau_{n-1}\right) D\left(f\left(\tau_{n-1}+k_{n}^{(s)}\right)\right)\right| \\
& +k_{n}^{(s-1)}\left|f_{\bar{\tau}}\left(\tau_{n}\right)\right| \cdot \mid D\left(G\left(y_{n}, 0 ; t_{n-1}+k_{n}^{(s)}, \tau_{n-1}\right) \mid\right]
\end{aligned}
$$

where

$$
\begin{aligned}
\Gamma_{1}\left(k_{n}^{(s)}\right)= & 1+a^{2} k_{n}^{(s)} G_{\xi}\left(y_{n}, \xi_{n} ; t_{n-1}+k_{n}^{(s)}, \tau_{n-1}\right), \\
\Gamma_{2}\left(k_{n}^{(s)}\right)= & \sum_{j=1}^{J+n-1} h G\left(y_{n}, \xi_{j} ; t_{n-1}+k_{n}^{(s)}, \tau_{n-1}\right) u_{\bar{\xi}}\left(\xi_{j}, \tau_{n-1}\right) \\
& -k_{n}^{(s)} G\left(y_{n}, 0 ; t_{n-1}+k_{n}^{(s)}, \tau_{n-1}\right) \cdot f_{\tau}\left(\tau_{n}\right) .
\end{aligned}
$$

It is easy to see that from Lemmas $2,3,4$, and (3.5)

(3.8) $\quad\left|\Gamma_{1}^{-1}\left(k_{n}^{(s-1)}\right)\right|<4$

(3.9) $\quad\left|\Gamma_{2}\left(k_{n}^{(s)}\right)\right|<2 \tilde{M} \quad$ (for small $h$ )

$$
\begin{gathered}
\left|G\left(y_{n}, 0 ; t_{n-1}+k_{n}^{(s)}, \tau_{n-1}\right) D\left(f\left(\tau_{n-1}+k_{n}^{(s)}\right)\right)\right| C_{1} \frac{\left|D\left(k_{n}^{(s)}\right)\right|}{\sqrt{h}} \\
\left(C_{1}=\frac{\sqrt{2 \kappa M} M_{1}}{a}\right),
\end{gathered}
$$

and from Lemma 5,

$$
\begin{aligned}
& \left|k_{n}^{(s-1)} f_{\tilde{r}}\left(\tau_{n}\right) D\left(G\left(y_{n}, 0 ; t_{n-1}+k_{n}^{(s)}, \tau_{n-1}\right)\right)\right| \\
& \quad<\frac{4 a^{2} k_{n}^{(s-1)} M_{1}}{\pi h^{3}} \int_{-\pi}^{\pi}\left(\Lambda^{(s)} \Lambda^{(s-1)}\right)^{-1} \sin ^{2} \frac{\omega}{2} d \omega\left|D\left(k_{n}^{(s)}\right)\right| \\
& \quad<\frac{a^{2} k_{n}^{(s-1)} M_{1}\left|D\left(k_{n}^{(s)}\right)\right|}{h^{3} \sqrt{\lambda_{n}^{(s)} \lambda_{n}^{(s-1)}}\left(\sqrt{\lambda_{n}^{(s)}}+\sqrt{\lambda_{n}^{(s-1)}}\right)} \\
& \quad<\frac{C_{1}}{\sqrt{h}} D\left(k_{n}^{(s)}\right),
\end{aligned}
$$

where 


$$
\begin{aligned}
& \Lambda^{(s)}=1+4 \lambda_{n}^{(s)} \sin ^{2} \frac{\omega}{2}, \quad \Lambda^{(s-1)}=1+4 \lambda_{n}^{(s-1)} \sin ^{2} \frac{\omega}{2}, \\
& \lambda_{n}^{(s)}=\frac{a^{2} k_{n}^{(s)}}{h^{2}}, \quad \lambda_{n}^{(s-1)}=\frac{a^{2} k_{n}^{(s-1)}}{h^{2}} .
\end{aligned}
$$

By the mean value theorem

$$
\begin{aligned}
\left|D\left(\Gamma_{1}^{-1}\left(k_{n}^{(s)}\right)\right)\right|< & 16 \mid\left[a^{2} G_{\xi}\left(y_{n}, \eta_{n} ; t_{n-1}+\bar{k}, \tau_{n-1}\right)\right. \\
& \left.+a^{2} \bar{k} \frac{d G}{d \bar{k}}\left(y_{n}, \eta_{n} ; t_{n-1}+\bar{k}, \tau_{n-1}\right)\right]|\cdot| D\left(k_{n}^{(s)}\right) \mid \\
< & \frac{16 a^{2}}{\pi h^{2}}\left[\int_{-\pi}^{\pi} \frac{\sin ^{2} \frac{\omega}{2} d \omega}{\left(1+4 \bar{\lambda} \sin ^{2} \frac{\omega}{2}\right)^{2}}\right. \\
& \left.+\left|\int_{-\pi}^{\pi} \frac{\sin \frac{\omega}{2} \sin \left\{2(J+n)-\frac{1}{2}\right\} \omega d \omega}{\left(1+4 \bar{\lambda} \sin ^{2} \frac{\omega}{2}\right)^{2}}\right|\right] \cdot\left|D\left(k_{n}^{(s)}\right)\right|
\end{aligned}
$$

where $\bar{k}$ is a value between $k_{n}^{(s)}$ and $k_{n}^{(s-1)}, \bar{\lambda}=\frac{a^{2} \bar{k}}{h^{2}}$. By (2.18) in Lemma 5 and (2.24) in Lemma 6,

$$
\left|D\left(\Gamma_{1}^{-1}\left(k_{n}^{(s)}\right)\right)\right|<\left[\frac{2}{a} \frac{h}{\bar{k}^{3 / 2}}+8(1+4 \sqrt{2}) a \frac{1}{\left\{2(J+n)-\frac{1}{2}\right\} h \sqrt{\bar{k}}}\right] \cdot\left|D\left(k_{n}^{(s)}\right)\right|
$$

$$
<C_{2} \frac{\left|D\left(k_{n}^{(s)}\right)\right|}{\sqrt{h}}, C_{2}=\frac{4 \sqrt{2}(\kappa M)^{3 / 2}}{a}+\frac{4(8+\sqrt{2}) a \sqrt{\kappa M}}{l} .
$$

Finally we consider the sum

$$
B \equiv \sum_{j=1}^{J+n-1} h D\left(G\left(y_{n}, \xi_{j} ; t_{n-1}+k_{n}^{(s)}, \tau_{n-1}\right)\right) u_{\bar{\xi}}\left(\xi_{j}, \tau_{n-1}\right) \text {. }
$$

Here

$$
\begin{aligned}
D\left(G\left(y_{n}, \xi_{j} ; t_{n-1}+k_{n}^{(s)}, \tau_{n-1}\right)\right) & \\
=-\frac{1}{2 \pi h} \int_{-\pi}^{\pi} \frac{4 a^{2}}{h^{2}} \sin ^{2} \frac{\omega}{2}\left(\Lambda^{(s)} \Lambda^{(s-1)}\right)^{-1}\left[e^{-i(J+n-j)_{\omega}}\right. & \left.\quad+e^{-i(J+n+j-1) \omega}\right] d \omega \cdot D\left(k_{n}^{(s)}\right) \\
& =a^{2} \Phi_{\xi \tilde{\xi}}\left(\xi_{j}\right) D\left(k_{n}^{(s)}\right),
\end{aligned}
$$


where

$$
\Phi\left(\xi_{j}\right)=\frac{1}{2 \pi h} \int_{-\pi}^{\pi}\left(\Lambda^{(s)} \Lambda^{(s-1)}\right)^{-1}\left[e^{-i(J+n-j) \omega}+e^{-i(J+n+j-1) \omega}\right] d \omega .
$$

Hence

$$
\begin{aligned}
& B=a^{2} D\left(k^{(s)}\right) \sum_{j=1}^{J+n-1} h \Phi_{\xi \bar{\xi}}\left(\xi_{j}\right) u_{\bar{\xi}}\left(\xi_{j}, \tau_{n-1}\right) \\
&=a^{2} D\left(k^{(s)}\right)\left[\Phi_{\xi}\left(\eta_{n-1}\right) u\left(\eta_{n-1}, \tau_{n-1}\right)-\Phi_{\xi}(0) u_{\bar{\xi}}\left(0, \tau_{n-1}\right)\right. \\
&\left.-\sum_{j=0}^{J+n-2} h \Phi_{\xi}\left(\xi_{j}\right) u_{\xi \bar{\xi}}\left(\xi_{j}, \tau_{n-1}\right)\right] .
\end{aligned}
$$

Thus

$$
\begin{aligned}
& |B|<a^{2}\left[\tilde{M}\left|\Phi_{\xi}\left(\eta_{n-1}\right)\right|+\tilde{M}\left|\Phi_{\xi}(0)\right|\right. \\
& \left.\quad+\frac{\bar{M}}{a^{2}} \sum_{j=0}^{J+n-2} h\left|\Phi_{\xi}\left(\xi_{j}\right)\right|\right] \cdot\left|D\left(k_{n}^{(s)}\right)\right| .
\end{aligned}
$$

Here

$$
\begin{aligned}
\Phi_{\xi}\left(\xi_{j}\right)= & -\frac{2}{\pi h^{2}} \int_{-\pi}^{\pi}\left\{\Lambda^{(s)} \Lambda^{(s-1)}\right\}^{-1} \sin ^{2} \frac{\omega}{2} \sin (J+n) \omega \sin j \omega d \omega \\
& +\frac{1}{2 \pi h^{2}} \int_{-\pi}^{\pi}\left\{\Lambda^{(s)} \Lambda^{(s-1)}\right\}^{-1} \sin \omega \sin (J+n-j) \omega d \omega \\
& -\frac{1}{2 \pi h^{2}} \int_{-\pi}^{\pi}\left\{\Lambda^{(s)} \Lambda^{(s-1)}\right\}^{-1} \sin \omega \sin (J+n+j) \omega d \omega
\end{aligned}
$$

and from Lemma 5 and Lemma 8

$$
\begin{aligned}
\left|\Phi_{\xi}\left(\xi_{j}\right)\right|< & \frac{h}{2 a^{3} \hat{k} \sqrt{\breve{k}}}+\frac{y_{n}+x_{j}}{4 a^{3} \hat{k}^{3 / 2}} \exp \left(-\frac{y_{n}+x_{j}}{a \sqrt{\hat{k}}}\right)+\frac{C_{3} h}{\hat{k} \sqrt{\check{k}}} \\
& +\left\{\begin{array}{l}
\left.\frac{y_{n}-x_{j}}{4 a^{3} \hat{k}^{3 / 2}} \exp \left(-\frac{y_{n}-x_{j}}{a \sqrt{\hat{k}}}\right)+\frac{C_{3} h}{\hat{k} \sqrt{\check{k}}} \quad \text { (for } y_{n}-x_{j}>3 a \sqrt{\hat{k}}\right) \\
\text { or alternatively } \\
\frac{C_{3}\left(y_{n}-x_{j}\right)}{\hat{k} \sqrt{\breve{k}}}
\end{array}\right.
\end{aligned}
$$


564

TAtsuo Nog

where $\hat{k}=\max \left(k_{n}^{(s)}, k_{n}^{(s-1)}\right), \check{k}=\min \left(k_{n}^{(s)}, k_{n}^{(s-1)}\right)$ and $C_{3}=\frac{\pi^{3}}{4 a^{3}}$.

Using (3.4) we have $\frac{h}{\hat{k} \sqrt{\breve{k}}}<\frac{(2 \kappa M)^{3 / 2}}{\sqrt{h}}$. Hence

$$
\begin{aligned}
& \left|\Phi_{\xi}\left(\xi_{j}\right)\right|<\frac{y_{n}+\xi_{j}}{4 a^{3} \hat{k}^{3 / 2}} \exp \left(-\frac{y_{n}+\xi_{j}}{a \sqrt{\hat{k}}}\right)+\frac{C_{4}}{\sqrt{h}} \\
& +\left\{\begin{array}{l}
\frac{y_{n}-\xi_{j}}{4 a^{3} \hat{k}^{3 / 2}} \exp \left(-\frac{y_{n}-\xi_{j}}{a \sqrt{\hat{k}}}\right) \quad\left(\text { for } y_{n}-\xi_{j}>3 a \sqrt{\hat{k}}\right) \\
\text { or alternatively } \\
\frac{C_{3}\left(y_{n}-\xi_{j}\right)}{\hat{k} \sqrt{\breve{k}}}
\end{array}\right. \\
& \left(C_{4}=\frac{\sqrt{2}(\kappa M)^{3 / 2}}{a^{3}}+2 C_{3}(\kappa M)^{3 / 2}\right) .
\end{aligned}
$$

In particular

$$
\left|\Phi_{\xi}\left(\eta_{n-1}\right)\right|<\frac{y_{n}+\eta_{n-1}}{4 a^{3} \hat{k}^{3 / 2}} \exp \left(-\frac{y_{n}+\eta_{n-1}}{a \sqrt{\hat{k}}}\right)+\frac{C_{5}}{\sqrt{h}}
$$

and

$$
\begin{aligned}
&\left|\Phi_{\xi}(0)\right|<\frac{y_{n}}{2 a^{3} \hat{k}^{3 / 2}} \exp \left(-\frac{y_{n}}{a \sqrt{\hat{k}}}\right)+\frac{C_{5}}{\sqrt{h}} \\
&\left(C_{5}=C_{4}+(2 \kappa M)^{3 / 2} C_{3}\right) .
\end{aligned}
$$

From (3.13)-(3.16) we have

$$
\begin{aligned}
|B|< & a^{2} \tilde{M}\left[\frac{y_{n}+\eta_{n-1}}{4 a^{3} \hat{k}^{3 / 2}} \exp \left(-\frac{y_{n}+\eta_{n-1}}{a \sqrt{\hat{k}}}\right)+\frac{y_{n}}{2 a^{3} \hat{k}^{3 / 2}} \exp \left(-\frac{y_{n}}{a \sqrt{\hat{k}}}\right)\right. \\
& \left.+\frac{2 C_{5}}{\sqrt{h}}\right] \cdot\left|D\left(k_{n}^{(s)}\right)\right|+\bar{M}\left[\sum_{j=0}^{J+n-2} \frac{y_{n}+\xi_{j}}{4 a^{3} \hat{k}^{3 / 2}} \exp \left(-\frac{y_{n}+\xi_{j}}{a \sqrt{\hat{k}}}\right)\right. \\
& +\frac{C_{4} \eta_{n-1}}{\sqrt{h}}+\sum_{j=0}^{J_{1}} h \frac{y_{n}-\xi_{j}}{4 a^{3} \hat{k}^{3 / 2}} \exp \left(-\frac{y_{n}-\xi_{j}}{a \sqrt{\hat{k}}}\right)+
\end{aligned}
$$




$$
\left.+C_{3} \sum_{j=J_{1}+1}^{J+n-2} h \frac{y_{n}-\xi_{j}}{\hat{k} \sqrt{\check{k}}}\right] \cdot\left|D\left(k_{n}^{(s)}\right)\right|,
$$

where $J_{1}=\max \left\{j ; y_{n}-\xi_{j}>3 a \sqrt{\hat{k}}\right\}$. Here we note that

$$
\begin{aligned}
& 2 l<y_{n}+\eta_{n-1}<2 l+2 M t_{n}, \\
& l<y_{n}<l+M t_{n} .
\end{aligned}
$$

It follows from (3.19) that

$$
\begin{aligned}
& |B|<a^{2} \tilde{M}\left[\frac{l+M t_{n}}{2 a^{3} \hat{k}^{3 / 2}}\left\{\left(\exp \left(-\frac{2 l}{a \sqrt{\hat{k}}}\right)+\exp \left(-\frac{l}{a \sqrt{\hat{k}}}\right)\right\}+\frac{2 C_{5}}{\sqrt{h}}\right] \cdot\left|D\left(k_{n}^{(s)}\right)\right|\right. \\
& +\bar{M}\left[\frac{1}{4 a^{3} \hat{k}^{3 / 2}} \int_{0}^{y_{n}}\left\{\left(y_{n}-x\right) \exp \left(-\frac{y_{n}-x}{a \sqrt{\hat{k}}}\right)+\left(y_{n}+x\right) \exp \left(-\frac{y_{n}+x}{a \sqrt{\hat{k}}}\right)\right\} d x\right. \\
& \left.+\frac{9 a^{2} C_{2}}{2 \sqrt{\breve{k}}}+\frac{C_{4}\left(l+M t_{n}\right)}{\sqrt{h}}\right] \cdot\left|D\left(k_{n}^{(s)}\right)\right|
\end{aligned}
$$

and further for small $h(\hat{k})$

$$
\begin{aligned}
& |B|<\left[\frac{4 a^{2} \tilde{M}}{\sqrt{h}}+\frac{\bar{M}}{2 a \sqrt{\hat{k}}} \int_{0}^{\infty} \sigma e^{-\sigma} d \sigma+\frac{9 a^{2} C_{2} \bar{M}}{2 \sqrt{\tilde{k}}}\right. \\
& \left.+\frac{C_{4}\left(l+M t_{n}\right) \bar{M}}{\sqrt{h}}\right] \cdot\left|D\left(k_{n}^{(s)}\right)\right|<\frac{C_{6}}{\sqrt{h}}\left|D\left(k_{n}^{(s)}\right)\right| \\
& \left(C_{6}=4 a^{2} \tilde{M}+\frac{\bar{M}}{\sqrt{2}}\left(\frac{1}{a}+9 a^{2} C_{3}\right) \sqrt{\kappa M}+C_{4}(l+M T) \bar{M}\right) .
\end{aligned}
$$

Consequently we get from (3.6), (3.8)-(3.11), (3.13)-(3.14) and (3.20)

$$
\begin{gathered}
\left|v_{n}^{(s)}-v_{n}^{(s-1)}\right|<\frac{C_{7}}{\sqrt{h}}\left|D\left(k_{n}^{(s)}\right)\right|, \\
C_{7}=2 \tilde{M} C_{2}+4\left(2 C_{1}+C_{6}\right) .
\end{gathered}
$$

Here we have from (1.17)

$$
\left|D\left(k_{n}^{(s)}\right)\right|<\frac{\kappa \sqrt{h}}{\beta}\left|v_{n}^{(s-1)}-v_{n}^{(s-2)}\right| .
$$


It follow from (3.21) and (3.22) that

$$
\left|v_{n}^{(s)}-v_{n}^{(s-1)}\right|<\frac{C_{7} \kappa}{\beta}\left|v_{n}^{(s-1)}-v_{n}^{(s-2)}\right| .
$$

If $\beta$ is chosen so large that

$$
\delta \equiv \frac{C_{7} \kappa}{\beta}<1
$$

then (3.2) holds with $\delta$ smaller than 1. Thus we have proved convergence of our iteration procedure.

\section{§4. Convergence of the Scheme as $h \rightarrow 0$}

We assume that

$$
\begin{aligned}
& \max _{0 \leqq t \leqq T}|f(t)|, \max _{0 \leqq x \leqq l}|\varphi(x)|<M_{0} \\
& \max _{0<t<T}|\dot{f}(t)|, \max _{0<x<l}|\dot{\varphi}(x)|<M_{1}
\end{aligned}
$$

and

$$
\max _{0<x<l}|\ddot{\varphi}(x)|<M_{2}
$$

By the maximum principle we have from (4.1)

$$
\max _{\substack{0 \leqq x_{j} \leqq y_{n} \\ 0<t_{n} \leqq T}}\left|u\left(x_{j}, t_{n}\right)\right|<M_{0}
$$

and also from the assumption $\varphi \leqq 0, f \leqq 0$,

$$
u\left(x_{j}, t_{n}\right) \leqq 0 .
$$

We shall see that it is sufficient for convergence proof to show a priori estimate

$$
\max _{t_{n}<T}\left|v_{n}\right|<M
$$

In fact we have then for small $h$

$$
\left|u_{\bar{t}}\left(y_{n-1}, t_{n}\right)\right|<2 \kappa M^{2}
$$


because

$$
0 \leqq u_{\bar{t}}\left(y_{n-1}, t_{n}\right)=-\frac{h}{k_{n}} v_{n}=-\frac{v_{n}}{2}\left(\sqrt{\kappa^{2} v_{n}^{2}+4 \beta \sqrt{h}}+\kappa v_{n}\right) .
$$

The system which $z=u_{\bar{t}}$ satisfies is

$$
\left\{\begin{array}{l}
a^{2} z_{x \bar{x}}\left(x_{j}, t_{n}\right)-z_{\bar{t}}\left(x_{j}, t_{n}\right)=0, \quad 0<x_{j}<y_{n-1}, \quad 0<t_{n}<T, \\
z\left(0, t_{n}\right)=f_{\bar{t}}\left(t_{n}\right), \quad 0<t_{n}<T, \\
z\left(y_{n-1}, t_{n}\right)=u_{\bar{t}}\left(y_{n-1}, t_{n}\right), \quad 0<t_{n}<T, \\
z\left(x_{j}, 0\right)=a^{2} \varphi_{x \bar{x}}\left(x_{j}\right), \quad 0<x_{j}<l .
\end{array}\right.
$$

By the maximum principle we get from (4.2), (4.3) and (4.7)

$$
\max _{\substack{0<x_{j}<y_{n} \\ t_{n}<T}}\left|u_{\bar{t}}\left(x_{j}, t_{n}\right)\right|<\bar{M}=\max \left\{M_{1}, a^{2} M_{2}, 2 \kappa M^{2}\right\}
$$

and

$$
\max _{\substack{0<x_{j}<y_{n} \\ t_{n}<T}}\left|u_{x \bar{x}}\left(x_{j}, t_{n}\right)\right|<\frac{M}{a^{2}} .
$$

Using the identity

$$
u_{x}\left(x_{j}, t_{n}\right)=v_{n}-\sum_{r=j+1}^{J+n-1} u_{x \bar{x}}\left(x_{r}, t_{n}\right) h
$$

and (4.6), (4.10) we obtain

$$
\max _{\substack{0<x_{j}<y_{n} \\ t_{n}<T}}\left|u_{x}\left(x_{j}, t_{n}\right)\right|<\tilde{M}=M+\frac{\bar{M}}{a^{2}}(l+2 \kappa M T) .
$$

We shall show convergence from a priori estimates (4.4), (4.6), (4.9)-(4.11). Let $h_{\alpha}$ tend to zero as $\alpha \rightarrow \infty$. From (1.10) corresponding $k_{n \alpha}$ tends to zero as $\alpha \rightarrow \infty$. Denote by $y_{\alpha}(t)$ the broken line crossing each right-endmesh-point $\left(y_{n}, t_{n}\right)$. Then we have from $(1.10)^{\prime},(4.6)$

$$
l \leqq y_{\alpha}(t) \leqq l+2 \kappa M T, \quad 0<t<T
$$

and also

$$
0<y_{\alpha}\left(t^{2}\right)-y_{\alpha}\left(t^{1}\right)<\kappa M\left(t^{2}-t^{1}\right), \quad 0<t^{1}<t^{2}<T
$$


Since last inequalities means that the sequence of functions $\left\{y_{\alpha}(t)\right\}$ is uniformly bounded and equi-continuous, it follows that there is a subsequence (which we denote again by $\left\{y_{\alpha}(t)\right\}$ which converges to a continuous function $y(t)$ uniformly in $0 \leqq t \leqq T$. The limit function satisfies by (4.12) and (4.13)

$$
\begin{gathered}
l \leqq y(t) \leqq l+2 M T, \quad 0<t<T \\
0<y\left(t^{2}\right)-y\left(t^{1}\right)<\kappa M\left(t^{2}-t^{1}\right), \quad 0<t^{1}<t^{2}<T .
\end{gathered}
$$

Let $u_{\alpha}$ be the solution of system (1.6)-(1.10) corresponding to $h_{\alpha}$. It is shown from (4.4), (4.6), (4.9)-(4.11) and (4.15) that a subsequence of $\left\{u_{\alpha}\right\}$ converges to the solution $u$ of (1.1)-(1.4) with the boundary $x=y(t)$ above defined uniformly in $0<x<y(t), 0<t<T$. (see Petrowsky [4])

We shall show that the pair of functions $(y(t), u(x, t))$ satisfies also the Stefan's condition (1.5) (hence all the system (1.1)-(1.5)). We can define $\left\{u_{\alpha}(x, t)\right\}$ for all $(x, t)$ extended from $\left\{u_{\alpha}\left(x_{j}, t_{n}\right)\right\}$ appropriately. Then we have from (4.10)

$$
\left|u_{\alpha \bar{x}}(x, t)-u_{\alpha \bar{x}}\left(x^{\prime}, t\right)\right|<\frac{\bar{M}}{a^{2}}\left|x-x^{\prime}\right|, \quad 0<t<T
$$

and

$$
\left|\frac{\partial u(x, t)}{\partial x}-\frac{\partial u\left(x^{\prime}, t\right)}{\partial x}\right|<\frac{\bar{M}}{a^{2}}\left|x-x^{\prime}\right|, \quad 0<t<T .
$$

Hence the limits

$$
\lim _{x \rightarrow y_{\alpha}(t)} u_{\alpha \bar{x}}(x, t)=v_{\alpha}(t) \quad \text { (uniformly in } t \text { ) }
$$

and

$$
\lim _{x \rightarrow y(t)} \frac{\partial u}{\partial x}(x, t)=v(t) \quad \text { (uniformly in } t \text { ) }
$$

exist. Consequently it follows from (4.18) and (4.19) that

$$
\lim _{\alpha \rightarrow \infty} v_{\alpha}(t)=v(t) \quad \text { (uniformly in } t \text { ). }
$$

By $(1.10)^{\prime}$ 


$$
\begin{aligned}
y_{\alpha}(t) & =l+\int_{0}^{t} \dot{y}_{\alpha}(t) d t=l+\sum k_{n} \frac{h}{k_{n}} \\
& =l+\frac{1}{2} \sum k_{n}\left(\sqrt{\kappa^{2} v_{\alpha n}^{2}+4 \beta \sqrt{h}}+\kappa v_{\alpha n}\right) .
\end{aligned}
$$

Here take $\alpha \rightarrow \infty$. Then we get by (4.20)

$$
y(t)=l+\kappa \int_{0}^{t} v(t) d t
$$

This means that $y(t)$ is differentiable and

$$
\dot{y}(t)=\kappa v(t)
$$

which is not but the Stefan's condition.

Since the solution of the system (1.1)-(1.5) is unique (Friedman [3]), it follows that not only a subsequence but also the full sequence $\left\{y_{\alpha}(t)\right\}$, $\left\{u_{\alpha}(x, t)\right\}$ themselves converge to $y(t)$ and $u(x, t)$ respectively.

It remains to show (4.6). Applying the formula (2.6) to the solution of (1.6)-(1.10) we have

$$
v_{n}=A_{1}^{-1}\left[A_{2}+A_{3}+A_{4}\right],
$$

where

$$
\begin{aligned}
& A_{1}=1+a^{2} k_{n} G_{\xi}\left(y_{n}, \eta_{n} ; t_{n}, \tau_{n-1}\right), \\
& A_{2}=\sum_{j=1}^{J} h G\left(y_{n}, \xi_{j} ; t_{n}, 0\right) \varphi_{\bar{\xi}}\left(\xi_{j}\right), \\
& A_{3}=-\sum_{P=1}^{n} k_{p} G\left(y_{n}, 0 ; t_{n}, \tau_{p-1}\right) f_{\tau}\left(\tau_{p}\right) \\
& A_{4}=-a^{2} \sum_{P=1}^{n} k_{p} G_{\xi}\left(y_{n}, \eta_{p} ; t_{n}, \tau_{p-1}\right) v_{p} .
\end{aligned}
$$

and

Directly from Lemmas 2 and 4 we get

$$
\left|A_{1}\right|<4
$$

$$
\left|A_{2}\right|<M_{1}
$$

By Lemma 3, 


$$
\left|A_{3}\right|<\frac{M_{1}}{a} \int_{0}^{t_{n}} \frac{d \tau}{\sqrt{t_{n}-\tau}}=\frac{2 M_{1}}{a} \sqrt{t_{n}} .
$$

Now we consider $A_{4}$ :

$$
\begin{aligned}
& A_{4}=A_{41}+A_{42}, \\
& A_{41}=-a^{2} \sum_{t_{n-\tau p-1}>\frac{2 h^{\frac{4}{3}}}{\sqrt{\beta}}} k_{p} G_{\xi}\left(y_{n}, \eta_{p} ; t_{n}, \tau_{p-1}\right) v_{p}, \\
& A_{42}=-a^{2} \sum_{t_{n-\tau p-1}<\frac{2 h \frac{3}{\sqrt{4}}}{\sqrt{\beta}}} k_{p} G_{\xi}\left(y_{n}, \eta_{p} ; t_{n}, \tau_{p-1}\right) v_{p} .
\end{aligned}
$$

First we have from (2.30)

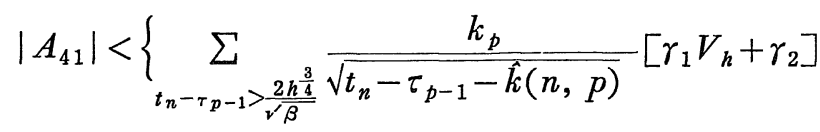

$$
\begin{aligned}
& \left.+\sum_{t_{n}-\tau_{p-1}>\frac{2 h \frac{3}{4}}{v^{\prime}}} \frac{\gamma_{3} \hat{k}(n, p)}{\left(t_{n}-\tau_{p-1}-\hat{k}_{p}(n, p)\right)^{\frac{3}{2}}}\right\}\|v\|
\end{aligned}
$$

where

$$
\begin{aligned}
& \|v\|=\max _{p=1, \ldots, n-1}\left|v_{p}\right| \\
& \gamma_{1}=\frac{2+\pi}{2 \sqrt{2 a}}, \quad \gamma_{2}=\frac{(1+2 \sqrt{2}) a}{4 l}, \quad r_{3}=\frac{a}{\sqrt{2} l}
\end{aligned}
$$

and $V_{h}$ is a bound for $\frac{h}{k_{p}}(p=1, \ldots, n-1)$ as in $\S 2$. Since $\hat{k}(n, p)<\frac{h^{\frac{3}{4}}}{\sqrt{\beta}}$,

$$
\left|A_{41}\right|<\left[\sqrt{2}\left(\gamma_{1} V_{h}+\gamma_{2}\right) \int_{0}^{t_{n-2}} \frac{d \tau}{\sqrt{t_{n}-\tau}}+\frac{2 \sqrt{2} \gamma_{3} h^{\frac{3}{4}}}{\sqrt{\beta}} \int_{0}^{t_{n}-\frac{2 h^{\frac{3}{4}}}{\sqrt{\beta}}} \frac{d \tau}{\left(t_{n}-\tau\right)^{\frac{3}{2}}}\right]\|v\|
$$

(4.27) $<\left[\left(\gamma_{1}^{\prime} V_{h}+\gamma_{2}^{\prime}\right) \sqrt{t_{n}}+\gamma_{3}^{\prime} h^{\frac{3}{8}}\right]\|v\|$

$$
\left(r_{1}^{\prime}=2 \sqrt{2} \gamma_{1}, \quad r_{2}^{\prime}=2 \sqrt{2} \gamma_{2}, \quad r_{3}^{\prime}=\frac{4 \gamma_{3}}{\beta^{\frac{1}{4}}}\right)
$$

Using (2.31) we have 


$$
\begin{gathered}
\left|A_{42}\right|<\left[\sum_{t_{n-\tau}-1<\frac{2 h^{\frac{3}{4}}}{v^{\prime}}}\left\{\frac{\gamma_{1} V_{h}}{\sqrt{t_{n}-\tau_{p-1}-\hat{k}(n, p)}}+\frac{\gamma_{4} V_{h}}{h} \sqrt{t_{n}-\tau_{p-1}}\right\} k_{p}\right]\|v\| \\
\left(\gamma_{4}=\frac{3 a}{4 l}\right) .
\end{gathered}
$$

Since $k_{q}>\frac{h}{k\|v\|}$ from $\left(1.10^{\prime}\right)$ we have

$$
\sqrt{t_{n}-\tau_{p-1}-\hat{k}(n, p)}>\sqrt{\frac{h}{\kappa\|v\|}} .
$$

Hence

$$
\begin{aligned}
\left|A_{42}\right|< & {\left[2 \gamma_{1} \sqrt{\frac{\kappa\|v\|}{\beta}} V_{h} h^{\frac{1}{4}}+\frac{\gamma_{4} V_{h}}{h} \int_{t_{n-\frac{2 h}{\gamma^{\prime}}} \frac{3}{4}}^{t_{n}} \sqrt{t_{n}-\tau} d \tau\right] } \\
= & {\left[r_{1}^{\prime \prime} h^{\frac{1}{4}} V_{h}\|v\|^{\frac{1}{2}}+\gamma_{4}^{\prime} h^{\frac{1}{8}} V_{h}\right]\|v\| } \\
& \left(\gamma_{1}^{\prime \prime}=2 \gamma_{1} \sqrt{\frac{\kappa}{\beta}}, \quad \gamma_{4}^{\prime}=\frac{2 \sqrt{2} \gamma_{4}}{3 \beta^{\frac{3}{4}}}\right) .
\end{aligned}
$$

We obtain from (4.26)-(4.28)

$$
\begin{gathered}
\left|A_{4}\right|<\left[\left(\gamma_{1}^{\prime} V_{h}+\gamma_{2}^{\prime}\right) \sqrt{t_{n}}+\gamma_{3}^{\prime} h^{\frac{3}{8}}+\gamma_{1}^{\prime \prime} h^{\frac{1}{4}} V_{h}\|v\|^{\frac{1}{2}}\right. \\
\left.+\gamma_{4}^{\prime} h^{\frac{1}{8}} V_{h}\right]\|v\| .
\end{gathered}
$$

Here

$$
V_{h}<\kappa\|v\|+\sqrt{\beta} h^{\frac{1}{4}}
$$

from $\left(1.10^{\prime}\right)$. Put

$$
\begin{aligned}
\left|A_{4}\right|< & {\left[\gamma_{3}^{\prime} h^{\frac{3}{8}}+\gamma_{4}^{\prime} \sqrt{\beta} h^{\frac{3}{8}}+\gamma_{1}^{\prime \prime} \sqrt{\beta} h^{\frac{1}{2}}\|v\|^{\frac{1}{2}}\right.} \\
& +\gamma_{4}^{\prime} \kappa h^{\frac{1}{8}}\|v\|+\gamma_{1}^{\prime \prime} \kappa h^{\frac{1}{4}}\|v\|^{\frac{3}{2}}+\left(\gamma_{2}^{\prime}+\gamma_{1}^{\prime} \sqrt{\beta} h^{\frac{1}{4}}\right. \\
& \left.\left.+\gamma_{1}^{\prime} \kappa\|v\|\right) \sqrt{t_{n}}\right]\|v\| \equiv L_{1}\left(\|v\|, t_{n}, h\right) .
\end{aligned}
$$


It follows from (4.23)-(4.25) and (4.29) that

$$
\left|v_{n}\right|<4\left[M_{1}+\frac{2 M_{1}}{a} \sqrt{t_{n}}+L_{1}\left(\|v\|, t_{n}, h\right)\right] .
$$

Put

$$
\hat{M}=4 M_{1}+1
$$

and take $h$ so small that

$$
\begin{aligned}
& \gamma_{2}^{\prime}+\gamma_{1}^{\prime} \sqrt{\beta} h^{\frac{1}{4}}+\gamma_{1}^{\prime} \kappa \hat{M}<2\left(\gamma_{2}^{\prime}+\gamma_{1}^{\prime} \kappa \hat{M}\right), \quad \text { and } \\
& \left(\gamma_{3}^{\prime} h^{\frac{3}{8}}+\gamma_{4}^{\prime} \sqrt{\beta} h^{\frac{3}{8}}+\gamma_{1}^{\prime \prime} \sqrt{\beta} \hat{M}^{\frac{1}{2}} h^{\frac{1}{2}}+\gamma_{4}^{\prime} \kappa \hat{M} h^{\frac{1}{8}}\right. \\
& \left.+\gamma_{1}^{\prime \prime} \kappa \hat{M}^{\frac{3}{2}} h^{\frac{1}{4}}\right) \hat{M}<\frac{1}{8}
\end{aligned}
$$

and take $\sigma$ so small that

$$
\left\{\frac{M_{1}}{a}+\left(\gamma_{2}^{\prime}+\gamma_{1}^{\prime} \kappa \hat{M}\right) \hat{M}\right\} \sqrt{\sigma}<\frac{1}{16}
$$

Then if we assume that

$$
\begin{aligned}
\|v\|= & \max _{p=1, \ldots, n-1}\left|v_{p}\right|<\hat{M} \\
& t_{n}<\sigma
\end{aligned}
$$

we have from (4.30) and (4.31)

$$
\left|v_{n}\right|<\hat{M} .
$$

Thus we get the local a priori estimate

$$
\left|v^{n}\right|<\hat{M} \quad \text { for } t_{n}<\sigma \text { and sufficiently small } h .
$$

Hence convergence follows from the last estimate for $0<t<\sigma$ as we noted above and existence of a local solution of the differential problem (1.1)(1.5) is established as a by-product. It is well known that global existence in our problem follows from local existence (see Friedman [3]). Therefore we have a priori estimate 


$$
\max _{\substack{0 \leqq x \leq y(t) \\ 0<t \leqq T}}\left|\frac{\partial u}{\partial x}(x, t)\right|<\hat{M}_{1}
$$

where $\hat{M}_{1}$ is a constant. From convergence properties as noted above we have for sufficiently small $h$

$$
\max _{\substack{0<x_{j} \leqq y_{n} \\ t_{n}<\sigma}}\left|u_{\bar{x}}\left(x_{j}, t_{n}\right)\right|<2 \hat{\bar{M}}_{1} \equiv M_{1} .
$$

Put

$$
M=4 \hat{\tilde{M}}_{1}+1
$$

and take $\sigma_{1}$ so small that

$$
\left\{\frac{\hat{\hat{M}}_{1}}{a}+\left(\gamma_{2}^{\prime}+\gamma_{1}^{\prime} \kappa M\right) M\right\} \sqrt{\sigma_{1}}<\frac{1}{16}
$$

(see (4.33)). Then we get

$$
\left|v\left(t_{n}\right)\right|<M \quad \text { for } 0<t_{n}<\sigma<\sigma_{1}
$$

as above. Here $\sigma_{1}$ is depending only on $M_{1}$, not on $t_{n}$. In the same way, we also have

$$
\left|v\left(t_{n}\right)\right|<M \quad \text { for } 0<t_{n}<\sigma+2 \sigma_{1}
$$

and so on. Thus we get a priori estimate

$$
\left|v\left(t_{n}\right)\right|<M \quad \text { for } 0<t_{n}<T
$$

which was desired.

\section{§5. Numerical Experiment}

We show some results of our numerical experiment using our difference scheme. We take, for example, the following data in the problem (1.1)(1.5):

$$
\begin{aligned}
& a=1.0, \quad l=1.0, \quad \kappa=1.0, \\
& f(t)=\left\{\begin{array}{cc}
-\cos \frac{\pi}{4} t, & 0<t<2 \\
0, & t>2
\end{array}\right.
\end{aligned}
$$


and

$$
\varphi(x)=x-1, \quad 0<x<1 .
$$

We solved this problem by the scheme (1.6)-(1.10) in the three cases:
i) $\beta=0.05, h=0.1$
ii) $\beta=0.05, h=0.01$
iii) $\beta=0, h=0.1$.

Here by $\beta=0$ we mean the case without the artificial heat flow term in (1.10), that is, we use the formula

$$
k_{n}^{(s+1)}=\frac{h}{\kappa v_{n}^{(s)}}
$$

instead of (1.17) in the iteration procedure. Fig. 1 shows the position of the free boundary in each case. In the third case the calculation could not be continued because the iteration determining $k_{7}$ did not converge.

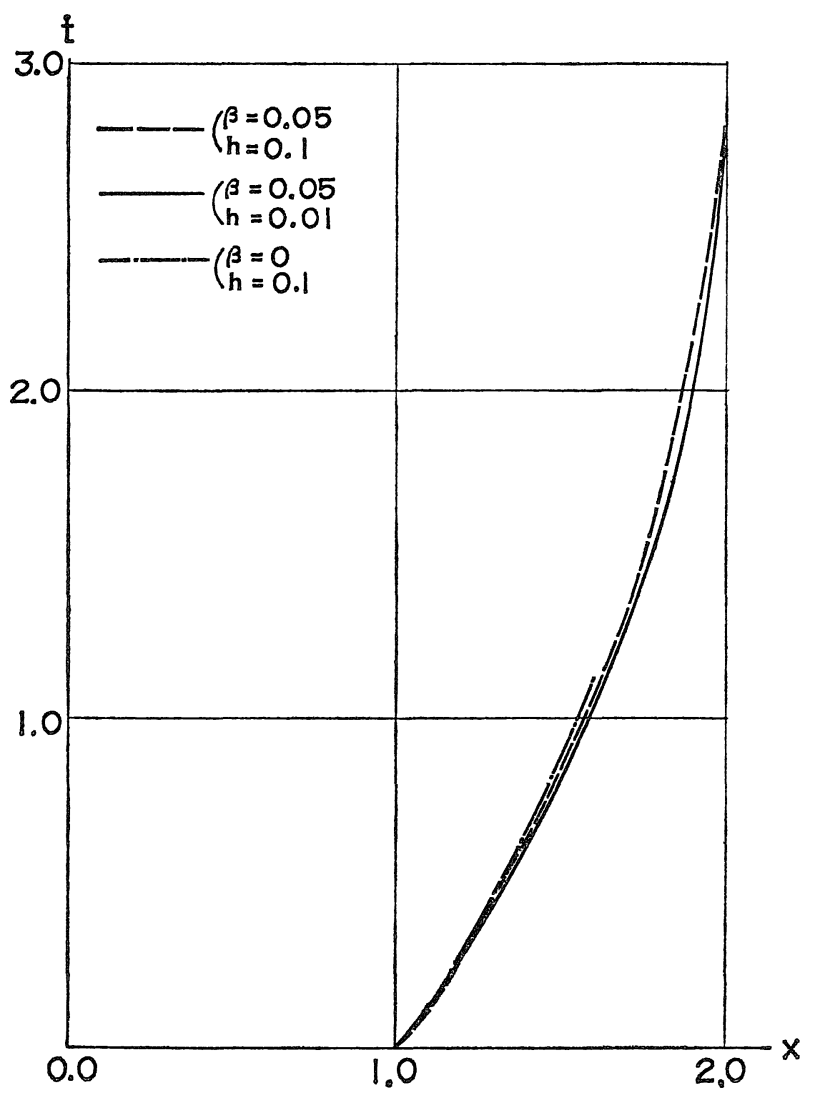

Fig. 1 
In the first two cases with artificial heat flow term the calculations could be done as far as we desired and the iteration at each time step converged within check bound $\left|k_{n}^{(s)}-k_{n}^{(s-1)}\right|<0.0001$ by $5 \sim 8$ times (the first case) or $3 \sim 4$ times (the second case). From comparison of the first two cases we know that even the first calculation rough mesh size $h=0.1$ shows sufficiently convergent feature.

\section{Acknowledgement}

The author deeply thanks Prof. K. Okugawa Prof. M. Yamaguti and our colleague participating in numerical analysis seminar at the Department of mathematics and physics of the Faculty of Engineering in Kyoto University for their criticisms and encouragements. And he is also indebted to Miss H. Shinoda for typewriting his manuscript.

\section{References}

[1] Douglas, J. Jr. and Gallie, T. M., On the numerical integration of a parabolic differential subject to a moving boundary condition, Duke Math. J., 22 (1955), 557-571.

[2] Vasilev, F.P., On finite difference methods for the solution of Stefan's singlephase problem, J. Comp. Math. and Math. Phys. 3 (1963), 861-873.

[3] Friedman, A., Free boundary problems for parabolic equations I. Melting of solids, J. Math. and Mech. 8 (1959), 499-518.

[4] Petrowsky, I.G., Lecture on partial differential equation, 1953.

[5] Rubinstein, L.I., Stefan's problem, «Zvaigzne》 publ., Riga, 1967. 
